Rev. 5, 1955, N.1; derselbe: The St. Lawrence Seaway and Urban Geography. Cornwall-Cardinal, Ontario. The Geogr. Rev. 45, 1955, N. 4; derselbe: The St. Lawrence Seaway and agricultural geography in the Cornwall-Cardinal area, Ontario. Geogr. Bulletin 8, 1956, Nr. 2-3. - W INKLER, E.: Kanadische New Towns. Plan 12, 1955, Nr. 11/12. - Hartley, H. R.: The Effects of the St. Lawrence Seaway on Grain Movements. Bloomington 1957. - Montreal Research Council, School of Commerce, McGill University: The Impact of the St. Lawrence Seaway on the Montreal Area. Montreal 1958. - BoEsch, H.: Die neue Wasserstraße in den nordamerikanischen Kontinent. Neue Zürcher Zeitung 180, 1959, Nr. 722. - HöffDing, W.: Die St. Lorenz-Wasserstraße. Neue Zürcher Zeitung 180, 1959, Nr. 1942, 2013. - Scotr, V. Taming the St. Lawrence. Discovery 20, 1959, Nr 6; Hills, T. L.: The St. Lawrence Seaway. London 1959. - Survey markets-Canada 1959. Montreal 1959. - Vgl. Canada Department of Mines and Technical Surveys, Geogr. Branch. Bibliographical Series. Ottawa $1950 \mathrm{ff}$.

\title{
THE ST. LAWRENCE SEAWAY
}

In the early summer 1959 the new St. Lawrence Seaway was opened, making the Great Lakes of North America accessible to the large ocean-going vessels of the world's seaports. After many decades of discussion between the USA and Canada work was finally begun on this enormous project and it was brought to completion in less than five years. The work consisted mainly in dredgeing out the waterway to a uniform depth of 10 yards and in the construction of new canals, particularly between the Lake Ontario and Montreal. Furthermore, the 31 smaller locks of the old waterway have been replaced by seven large locks. In addition, a vast hydro-electric power complex was built which will develop up to 1900000 kilowatts of electricity. The project required a large number of changes in the landscape, which probably will become even more prominent in future. For the economic life and culture of both neighbouring states, Canada and the USA, the «Main Street of North America» represents not only a vital line of communication but a symbol of international solidarity.

Der Verfasser dankt der Kanadischen Gesandtschaft in der Schweiz, Bern, bestens für die freundliche Überlassung von Photos aus dem National Film Board in Ottawa.

\section{LANGADI, ATYA-PATYA, SOWIE EINIGE VERWANDTE SPIELE DES FERNEN OSTENS*}

\author{
Paul G. Brewster
}

Eines der volkstümlichsten der in Bombay und Umgebung geübten Spiele ist Langadi, an welchem in der Regel nur Knaben teilnehmen. In diesem Fang-Spiel gelten die folgenden Hauptregeln:

1. Als Spielfeld dient ein Kreis von dreißig Fuß im Durchmesser. Innerhalb des Kreises befinden sich die Läufer und außerhalb desselben die Jäger. Sobald der Spielleiter pfeift, tritt einer der Jäger, auf einem Fuße hüpfend, in den Kreis und berührt so viele der Läufer als er vermag. Jeder, der vom Jäger berührt wurde, muß den Kreis sofort verlassen.

2. Es ist dem Jäger unter keinen Umständen erlaubt, beide Füsse auf den Boden zu setzen oder mit dem andern Fusse weiterzuhüpfen. So muß er, wenn er auf dem rechten $F u ß z u$ hüpfen begonnen hat, auf demselben weiterhüpfen, bis er erschöpft ist, oder bis aus einem anderen Grund der nächste Jäger seinen Platz einnehmen will. Verliert der Jäger das Gleichgewicht, so darf er sich dadurch zu helfen suchen, daß er beide Handflächen auf den Boden stützt. Wenn der Jäger nach dem Berühren eines Läufers das Gleichgewicht verliert und den andern Fuß auf den Boden setzt, so muß dieser Läufer den Kreis nicht verlassen. Kein anderer Körperteil des Jägers außer dem einen $\mathrm{Fu} \beta$ und den beiden Handflächen dürfen den Boden berühren, im andern Falle ist der nächste Jäger an der Reihe.

* Die Beschreibung der indischen Spiele verdanke ich der Freundlichkeit von Frl. Jaya S. Patel, Bombay, die Übersetzung ins Deutsche Hrn. dipl. Geogr. Arthur Dürst, Zürich. 
3. Die Jäger sollen in gleichen Abständen am Umfang des Kreises stehen und denselben in vorher festgelegter Reihenfolge betreten. Sie können auch an einem Haufen stehen und, einer nach dem andern, von einer bestimmten Stelle aus in den Kreis eintreten. Die letztere Anordnung ist für die Läufer einfacher, die im andern Falle die Reihenfolge der angreifenden Jäger vergessen könnten.

4. Der Läufer muß den Kreis verlassen, wenn er den Jäger absichtlich oder unabsichtlich berührt, oder wenn er den Kreis verläßt, um der Gefangennahme zu entgehen.

5. Der Jäger darf den Kreis straflos verlassen, solange er den andern Fuß nicht absetzt.

6. Dem Jäger ist es nur gestattet, sich auf die beiden Handflächen abzustützen, um sein verlorenes Gleichgewicht wiederzuerlangen, nicht aber um sich auszuruhen.

Zusätzlich zu diesen Hauptregeln, welche den Hergang des Spieles bestimmen, gibt es noch einige weitergehende Regeln wie die folgenden:

1. Jede Partei sollte neun Spieler haben.

2. Jede Partei ist zweimal an der Reihe, dazwischen werden fünf Minuten Pause eingeschaltet.

3. Wenn alle Spieler auf der Seite der Jäger zum Einsatz gekommen sind, und wenn es ihnen nicht gelang, alle Läufer zu fangen, erhält die Mannschaft der Läufer zwei Punkte für jeden Spieler, welcher den Kreis nicht verlassen mußte.

4. Wenn anderseits alle Läufer den Kreis verlassen mußten, jedoch einige der Jäger noch nicht an der Reihe waren, erhält die Partei der Jäger zwei Punkte für jeden Spieler, der nicht zum Einsatz kam.

5. Die Spieldauer sollte fünf Minuten oder länger betragen; sie wird zu Beginn des Spieles ausgemacht. Wenn keine der beiden Parteien innerhalb dieser Zeit alle Spieler verloren bzw. eingesetzt hat, erhält jede Partei für jeden Spieler, der sich vor der Gefangennahme retten konnte bzw. der nicht als Jäger zum Einsatz kam, zwei Punkte.

6. Es sollte ein Schiedsrichter vorhanden sein, der dafür sorgt, daß die Spielregeln befolgt werden und der die Resultate der beiden Parteien notiert.

Eine Variante des genannten Spieles ist Bethi Langadi. Es wird gleich gespielt mit Ausnahme der folgenden Änderungen:

1. Alle Teilnehmer spielen mit am Handgelenk zusammengebundenen Armen und müssen in kauernder Stellung hüpfen.

2. Der Jäger muß den Kopf des Läufers mit seinem eigenen Kopf berühren. Das Berühren eines andern oder mit einem andern Körperteil ist verboten.

3. Fällt einer der Läufer und löst sich dabei die Fesselung der Hände, muß er den Kreis nicht verlassen.

4. Der Läufer muß den Kreis verlassen, wenn a) sich die Fesselung seiner Hände löst oder wenn er sich während des Hüpfens mehr als halb aufrichtet, b) irgend ein anderer Körperteil den Boden berührt, c) er den Kreis verläßt oder d) absichtlich irgend einen Körperteil des Jägers berührt.

5. Der Jäger darf nicht mehr weiterspielen, wenn irgend ein Körperteil mit Ausnahme des Fusses den Boden berührt, oder wenn sich die Fesselung seiner Hände löst. Es ist ihm jedoch gestattet, den Kreis zu verlassen.

6. Es ist dem Jäger nicht gestattet, sich auszuruhen.

Weitere Beschränkungen, wie sie den Teilnehmern in Oobhi Langadi, einer andern Variante auferlegt werden, gestalten die Ausführung des Spieles immer schwieriger. Beinen.

1. Alle Teilnehmer, sowohl Läufer wie Jäger spielen mit zusammengebundenen

2. Der Läufer muß das Spiel verlassen wenn er a) über den Kreis hinausgeht oder irgend ein Körperteil mit Ausnahme der Füße den Boden berührt, b) sich die Fesselung der Füße löst oder c) er den Jäger zu Fall zu bringen oder ihn sonstwie zu hindern sucht. 


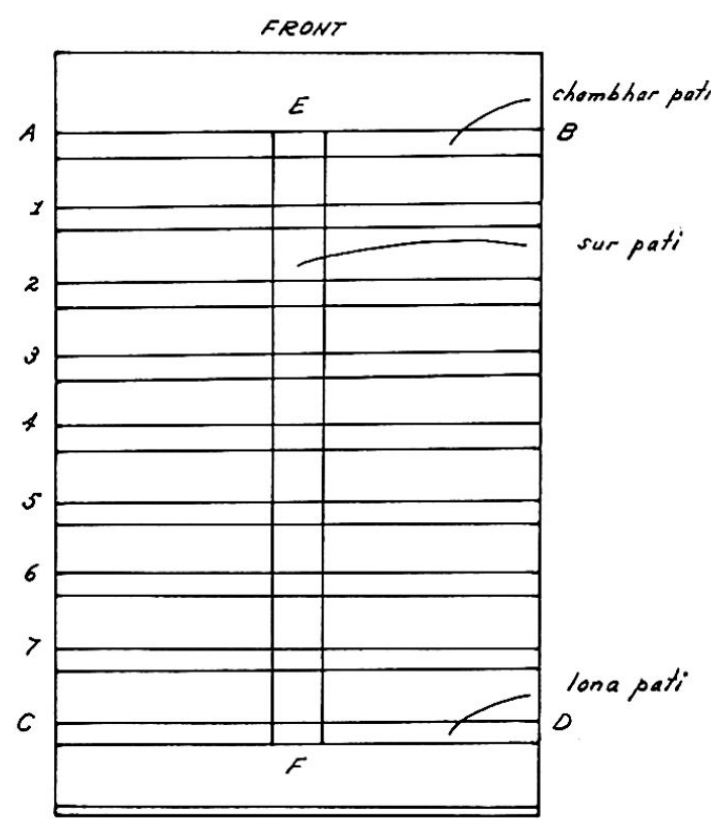

Fig. 1 Atya-Patya-Spiel

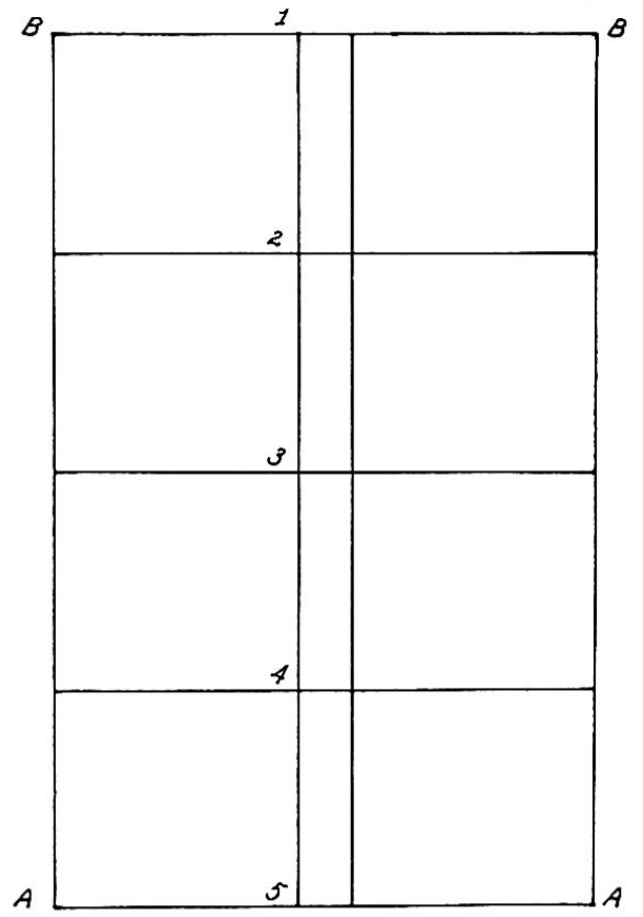

Fig. 2 Atipati oder Lonpat-Spiel

3. Löst sich die Fesselung der Beine des Jägers, so muß er das Spiel verlassen.

4. Es ist dem Jäger nicht gestattet, sich auszuruhen ${ }^{1}$.

Das Atya-Patya-Spiel, manchmal auch als Atipata oder Lonpat bekannt (Fig. 1 und 2) wird ebenfalls von zwei Parteien von je neun Spielern ausgeführt und geht über zwei Spielzeiten von je sieben Minuten Dauer. Das Spielfeld ist viereckig und wird in der Mitte durch einen in der Länge verlaufenden Streifen, sur pati genannt, und durch quer dazu verlaufende Streifen, die patis, abgeteilt (pati = Streifen). Der vorderste der letzteren ist als chambhar pati (chambhar $=$ Schuster) ${ }^{2}$, der letzte als lona pati bekannt. Eine parallele Linie wird vor der chambhar pati und eine entsprechende hinter der lona pati gezogen ${ }^{3}$.

Das Spiel geht wie folgt vor sich: Die Anführer der Parteien ziehen das Los und der Gewinner entscheidet, ob er die patis verteidigen oder queren will. Der Anführer der Verteidiger stellt einen Spieler in jede der patis; diese Spieler heißen patiwalla. Die chambhar pati sowie die sur pati sind beide nur durch einen einzigen Spieler bewacht; er wird sur genannt.

Aufgabe der andern Partei ist es, die patis einer nach dem andern zu durchqueren, ohne von den patiwallas berührt zu werden. Gelingt es einem Spieler alle patis, inbegriffen die lona pati zu durchqueren, so macht er sich auf den Rückweg. Er versetzt nun dem patiwalla der lona pati einen leichten Schlag und dieser muß sich sofort

1 In einer andern Variante des Spieles laufen die Jäger paarweise, indem ein Bein des einen mit einem Bein des andern zusammengebunden wird. In diesem Falle mißt der Kreis gewöhnlich $24 \mathrm{Fu}$ im Durchmesser anstatt $30 \mathrm{Fu}$. Neuerdings neigt man dazu, ein quadratisches Feld von $30 \times 30 \mathrm{Fu}$ zu benützen anstatt des kreisförmigen.

Langadi ist vor allem in den Schulen von Bombay sehr volkstümlich. Variationen der allgemeinen Form werden selten gespielt.

2 Jede pati ist $23 \mathrm{Fu}$ lang und $13 \mathrm{Fu}$ breit. Das Spielfeld mißt $89 \mathrm{Fu}$ in der Länge.

${ }^{3}$ Für eine Beschreibung des Spieles, wie es durch die Balahi gespielt wird, vergleiche man Stephen Fuchs: The Children of Hari: a Study of the Nimar Balahis in the Central Provinces of India. Vienna 1950, pp. 124-125. Hier wird es während der heißen Jahreszeit in Mondscheinnächten von Knaben und Mädchen gespielt (jedoch getrennt). Die Wächter, welche auf den letzten vier Linien aufgestellt sind, dürfen ihren Standort nicht verlassen, aber der auf der ersten Querlinie eingesetzte Spieler kann auch die lange Mittellinie, wie, sofern nötig, auch jede der andern Querlinien benützen. 
umdrehen und sich dem Spieler zuwenden. Beim Erreichen der nächsten pati versetzt er dem zweiten patiwalla einen Schlag, der sich ebenfalls umdreht, und so weiter. Kommt einer der Spieler, nachdem er alle patis durchquert und den Rückweg gemacht hat, ohne eine der Regeln zu verletzen, zum Ausgangspunkt zurück, so bedeutet das, daß seiner Partei ein lona (180 Punkte) gutgeschrieben wird. Wurde kein lona erzielt, erhält die Partei zehnmal die Zahl der patis, die der beste Spieler (pökka - Spieler genannt) zu überqueren vermochte, an Punkten.

Die Spieler, welche die angreifende Partei bilden dürfen nicht außerhalb des Feldes gehen. Die patiwallas ihrerseits dürfen nur längs der patis laufen. Hat das Spiel einmal begonnen, so ist es ihnen nicht gestattet die pati zu wechseln und es ist ihnen ebenfalls nicht gestattet, sich umzudrehen, bis ihnen der zurückkehrende Spieler auf den Rücken geschlagen hat. Sobald sie sich umgedreht haben, ist es ihnen wiederum nicht mehr erlaubt, sich zurückzuwenden.

Berührt der patiwalla einen der Spieler, wodurch dieser gefangen ist, muß der patiwalla dabei mit beiden Füßen den Boden berühren; ist einer davon abgehoben, so muß der berührte Spieler das Spielfeld nicht verlassen.

Die Partei mit der höchsten erzielten Punktzahl ist der Gewinner.

Nach der Beschreibung zu urteilen, welche Majumdar gegeben hat, gleicht das Chhur-Spiel dem eben beschriebenen. Im Chhtur-Spiel wechseln die Parteien jedoch ihre Rollen, wenn einer der Angreifer während des Versuches, die patis zu queren, gefangen wird; die Seite der Angreifer erhält dann keine Punkte. Nach dem Autor ist das Spiel ähnlich dem Daria-bandha, welches in den Dörfern von Bengal gespielt wird 4.

Das singhalesische Spiel Thattu wird ebenfalls auf einem rechteckigen Spielfeld gespielt, welches durch längs und quer verlaufende Linien in Quadrate aufgeteilt ist. Eine der beiden Parteien versucht, das Rechteck zu durchqueren, während es die andere Partei bewacht. Unter den Verteidigern ist ein «Wanderer», der längs allen Linien laufen darf, mit Ausnahme der langen seitlichen. Wächter, welche an jeder querlaufenden Linie aufgestellt sind, hindern die eindringende Partei, dieselben zu überqueren indem sie deren Spielern einen Schlag versetzen. Die Partei, welche die größere Anzahl von Spielern aufweist, die das Feld unbehelligt überqueren konnten, ist Sieger ${ }^{5}$.

Das rechteckige Feld, welches für die Ausführung des burmesischen Spieles Salts (Fig. 3) verwendet wird, richtet sich in der Größe nach dem verfügbaren Raum und bis zu einem gewissen Grade auch nach der Größe der teilnehmenden Spieler. Figur 3 zeigt ein Spielfeld aufgezeichnet für zehn Spieler, wovon fünf auf jeder Seite. Im Minimum können vier Spieler, im Maximum zehn teilnehmen.

Die Spieler 6 bis 10 bilden die angreifende Partei, welche das Feld, Quadrat für Quadrat bis ans entgegengesetzte Ende desselben queren und wieder zum Ausgangspunkt zurückkehren müssen, ohne von den Spielern 1-3 berührt worden zu sein und ohne die Linien $a-e$ und $e-r$ zu übertreten. Jeder Spieler bewacht eine Linie, jedoch ist der Spieler Nr. 1 nicht nur für die Linie $a-e$ verantwortlich, sondern er muß auch dem Spieler Nr. 2 zu Hilfe eilen, sofern derselbe diese benötigt. Spieler Nr. 1 ist der einzige Spieler, welcher sich nach rückwärts bewegen kann, um eine Linie zu verteidigen. Die Verteidiger, deren Räume bereits genommen wurden, stehen oder sitzen auf einer Seite und warten auf die Rückkehr der durchgedrungenen Angreifer.

Die Regeln, die festsetzen, unter welchen Umständen der Gegner als gefangen gilt, sind nicht einheitlich. Einige Spieler lassen das Berühren der Hand oder eines andern

${ }^{4}$ D. N. Majumdar: Some Outdoor and Sedentary Games of the Hos of Kolhan, Man in India. V, 3-4, 1925, 193.

5 P.E.P.Deraniyagala: Some Sinhala Combative, Field and Aquatic Sports and Games. Colombo: National Museums of Ceylon, 1951, p. 32. 


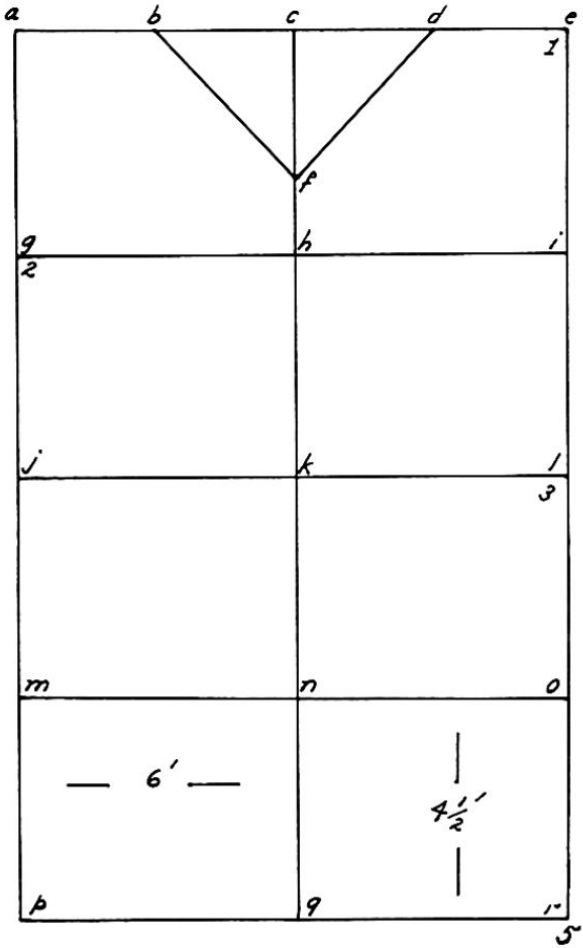

Fig. 3 Salts (burmesisch)

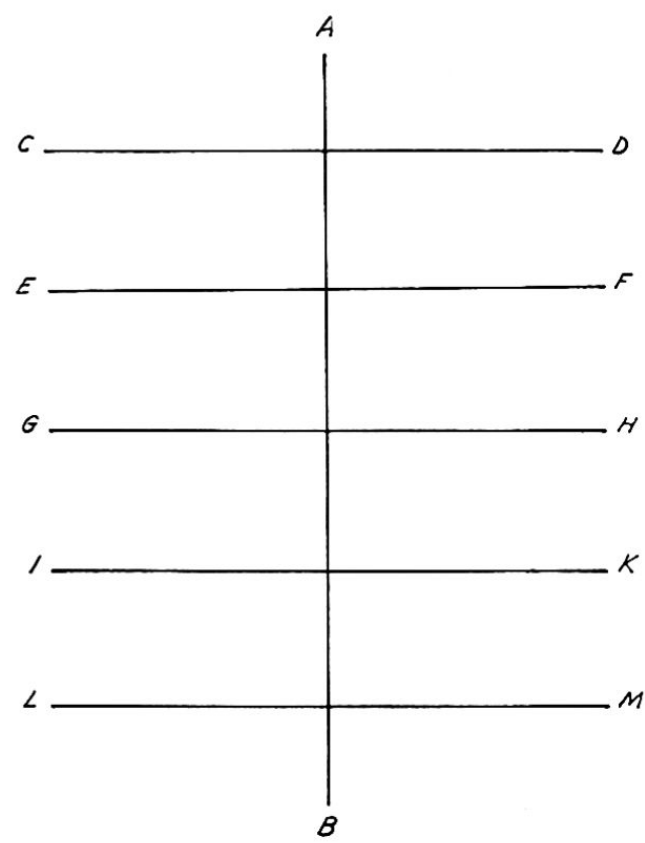

Fig. 4 Meuta'tham euë galah

unbedeckten Körperteils (mit Ausnahme des Kopfes oder des Gesichtes) gelten, während das Berühren eines losen Kleidungsstückes als ungültig betrachtet wird. Andere sind der Ansicht, daß jede Berührung welche vom Betroffenen gefühlt wird, Gefangennahme bedeutet ${ }^{5}$.

In einigen Varianten des Spieles endet eine Runde, wenn es einem Spieler gelungen ist das Spielfeld der Länge nach und wieder zurück zum Ausgangspunkt zu durchqueren ohne gefangengenommen zu werden. In anderen wird das Spiel solange weitergeführt, bis alle Angreifer durchgekommen sind oder gefangen wurden ${ }^{6}$.

Das malayische Gegenstück zum Atya-Patya ist das Tui-Spiel (manchmal auch Galah genannt). Gewöhnlich beträgt die Zahl der Spieler zehn und das Spielfeld wird ähnlich wie Fig. 4, nämlich wie folgt aufgezeichnet: Sofern sich zwölf Spieler beteiligen, werden statt vier Abteilungen deren fünf gemacht. Die Rollen der beiden Parteien werden durch das Los bestimmt.

Die Mittellinie, welche unter dem Namen galah panjang (der lange Pfahl) bekannt ist, wird vom Spielführer der verteidigenden Mannschaft bewacht, welcher allein für die Linie $A-B$ verantwortlich ist, aber auch Spieler, sofern nötig auf der Linie $C-D$ fangen kann. Gelingt es einem der Verteidiger, einen der Angreifer zu berühren, so wechseln die beiden Parteien ihre Rollen. Die Ersteren dürfen sich nicht von den Linien entfernen. Fangen die einen Angreifer, während ihre Füsse nicht mehr auf der Linie stehen, so gilt der Fang nicht. Diese Regel gilt gleichermaßen für den Spielführer.

Gelingt es einem der Angreifer, in irgend eines der Felder zu gelangen und bis $M$ durchzukommen ohne von einem der Verteidiger berührt zu werden, so ruft er «Tui, tui» und die übrigen Spieler seiner Partei rufen mit. Dann marschieren sie im Triumphzug nach $N$ hinunter. Am Schlusse dieses Triumphzuges nehmen sie wiederum ihre Rolle als angreifende Partei ein ${ }^{7}$.

${ }^{6}$ R. B. Dennis: Games and Children's Play (reprint from the Journal of the Burma Research Society, Rangoon, o. J.), p. 45.

7 D. F. A. HeRveY: Malay Games Journal of the Royal Anthropological Institute, XXXIII, 1903, 287. Eine kurze Beschreibung findet sich auch in W. W. SkEat: Malay Magic: An Introduction to the Folklore and Popular Religion of the Malay Peninsula. London, 1900, p. 500. 
Das achehnesische ${ }^{8}$ meuta'tham euë galah, (Fig. 4) eine Variante des galah panjang der malayischen Halbinsel wird in einem Feld gespielt, das dem letzteren ähnlich sieht aber ohne die Linien am Ende und auf den Seiten. Die Linie $A-B$ wird euë galah genannt; die Linien, welche dieselbe rechtwinklig schneiden sind die euë linetuëng. Die Zahl der letzteren hängt von der Zahl der teilnehmenden Spieler ab.

Jede euë wird von einem Spieler bewacht, und diese Wächter (sechs in der beigegebenen Figur) bilden eine Partei. Die Spieler der andern Partei versuchen von einer Stelle der Linie $L-M$ nach einem Punkt hinter der Linie $C-D$ zu gelangen.

Jeder Angreifer, der von einem Wächter berührt wird ist «tot» (das heißt er muß das Spielfeld verlassen) und nun ist seine Partei an der Reihe das Feld zu verteidigen. Die Wächter der quer verlaufenden Linien dürfen nur in der Richtung schlagen von welcher die Angreifer herkommen; der Verteidiger der Hauptlinie $(A-B)$ darf in jeder Richtung schlagen. Um einen Angreifer zu schlagen darf sich der Verteidiger nicht weiter von seiner Linie entfernen als er mit geschlossenen Füßen zu springen vermag. Gelingt es einem der angreifenden Partei von $L-M$ nach $C-D$ und wieder zurück zu gelangen ohne berührt zu werden so nennt man dies bilon und seine Partei hat die Runde gewonnen ${ }^{9}$.

${ }^{8}$ Gemeint ist damit das Volk der Atjeher im Norden Sumatras.

${ }^{9}$ C. Snouck Hurgrınje: The Achehnese (trans. A.W. S. O'Sullivan), London, 1906, II, 199.

\title{
LANGADI, ATYA-PATYA AND SOME ANALOGOUS GAMES FROM THE FAR EAST
}

This paper gives the rules for some very popular games of the Far East: Langadi, a kind of 'tag', played in the Bombay region, Atya-Patya sometimes known as Atipata or Lonpat, Chhur wich somewhat resembles the former, and Dãria - bãndha, played in Bengal villages. Then follow the Singhalese game Thattu and the Burmese Salts. A Malayan counterpart of Atya-Patya is the game Tui, sometimes called Galah. It has a variant played in the Peninsular Malays, the Achehnese meuta'tham euë galah.

\section{DAS PROBLEM DER GEOGRAPHISCHEN LANDSCHAFT}

\author{
ENDRE SZAVA-KOVATS
}

Es ist kein Zufall, daß sich heute die Theorie fast aller Wissenschaften intensiv mit der Überprüfung der Grundlagen beschäftigt. Soweit beurteilbar, ist ihr Zustand meist kritisch. Den Anzeichen nach beschränkte sich das «Erdbeben» des wissenschaftlichen Erkennens, dessen Epizentrum unzweifelbar im Grenzbereich der physikalischen und biologischen Disziplinen lag, in seiner Auswirkung nicht auf die benachbarten Gebiete, und die Häufigkeit der Nachbeben erscheint die Ansicht zahlreicher Beobachter zu rechtfertigen, wonach wir in eine neue Epoche eines erkenntnisgeschichtlichen Vulkanismus eingetreten sind. Auch die geographische Wissenschaft untersucht derzeit ihre grundlegenden Fragen. Ihr Interesse wandte sich diesen indes nicht erst jetzt zu: ihr Dasein war von jeher ein unaufhörlicher Kampf gegen die unablässige Umnebelung ihres Wesens. Es gab dabei einige Grundbegriffe, ohne die man sich die Geographie weder im Osten noch im Westen vorstellen kann, die aber dort wie da durch ihren Gebrauch fraglich geworden sind. Unter ihnen steht nach seiner Bedeutung der Begriff der geographischen Landschaft einzig da. Seine Wichtigkeit beruht indes nur auf einer großen Mehrheit von Meinungen, die das Bestehen der Geographie auf ihm zu gründen suchten, ohne ihn je hinreichend definiert zu haben. So konnte das Problem 